DOI: $10.21105 /$ joss.03437

\title{
grapesAgri1: Collection of Shiny Apps for Data Analysis in Agriculture
}

\author{
Pratheesh P. Gopinath ${ }^{1}$, Rajender Parsad ${ }^{2}$, Brigit Joseph ${ }^{1}$, and Adarsh \\ V. S. ${ }^{3}$
}

1 Department of Agricultural Statistics, College of Agriculture, Vellayani, Kerala Agricultural University, Kerala, India. 2 ICAR-Indian Agricultural Statistics Research Institute, New Delhi, India. 3 Department of Agricultural Statistics, BCKV, West Bengal, India

\section{Software}

- Review 七

- Repository ๘

\section{Summary}

- Archive ${ }^{\top}$

Editor: Fabian Scheipl ¿ Reviewers:

- Qadithirgis

- Qelimillera

Submitted: 10 June 2021 Published: 18 July 2021

\section{License}

Authors of papers retain copyright and release the work under a Creative Commons Attribution 4.0 International License (CC BY 4.0).
Agricultural experiments demand a wide range of statistical tools for analysis, which includes exploratory analysis, design of experiments, and statistical genetics. It is a challenge for scientists and students to find a suitable platform for data analysis and publish the research outputs in quality journals. Most of the software available for data analysis are proprietary or lack a simple user interface, for example $S A S \circledR$ is available in ICAR (Indian Council of Agricultural Research) for data analysis, though it is a highly advanced statistical analysis platform, and its complexity holds back students and researchers from using it. Some web applications like WASP (https://ccari.res.in/waspnew.html) and OPSTAT (http://14.139. 232.166/opstat/) used by the agricultural research community are user friendly but these applications don't provide options to generate plots and graphs.

The open source programming language $\mathrm{R}$ and associated ecosystem of packages, provides an excellent platform for data analysis but as of yet, is not heavily utilised by researchers in agricultural disciplines. Insufficient programming and computational knowledge are the primary challenges for agricultural researchers using $\mathrm{R}$ for analysis, as well as a preferences for researchers in agriculture to prefer a graphical user interface.

Efforts should therefore be made to develop a high quality, reliable open-source platform with a simple interactive user interface for data analysis in agriculture. Utilising the features of shiny package in $\mathrm{R}$, we have developed a collection of shiny apps for agricultural research called grapesAgri1 (General R shiny based Analysis Platform Empowered by Statistics for data analysis in Agriculture-part1). grapesAgri1 is hosted on the web (http://www.kaugrapes. com), downloadable as a standalone application, and now released as an $\mathrm{R}$ package.

By calling the functions in the grapesAgri1 package, a graphical user interface will open. Apps are self-explanatory and work by uploading files in CSV format. Results can be downloaded in a HTML format. Plots and graphs can be generated, which are also downloadable as .png files.

\section{Statement of need}

India has one of the world's largest agricultural education systems. According to the Indian Council of Agricultural Research (ICAR), the main regulator of agricultural education in India, there are 63 State Agricultural Universities, 4 deemed universities, and 3 Central Agricultural Universities. These institutions enroll annually about 15,000 students in undergraduate programs and over 7,000 students in post graduate programs and more than 2000 at PhD 
level. At any point, there are over 75,000 students studying in these institutions. Research activities are performed actively in all these universities. grapesAgri1 not only serves as a platform for data analysis but also can be used as a teaching tool in agricultural statistics. grapesAgri1 includes some basic statistical tools which were covered in the syllabi of undergraduate programs as well as in post graduate programs.

\section{Information for Users}

grapesAgri 1 is a collection of six shiny applications. Details of six applications are given below. Model dataset for testing can be downloaded from the main window of the application. Details for preparing CSV files are also included in the main window. Users just need to click on the browse button and upload the CSV file for analysis.

\section{Apps included in the package}

\begin{tabular}{|c|c|c|c|}
\hline SI. No. & App Title & Function to call & Utility \\
\hline 1 & $\begin{array}{l}\text { Descriptive Statistics } \\
\text { and Visualization }\end{array}$ & $\operatorname{descApp}()$ & $\begin{array}{l}\text { Summary statistics, summary } \\
\text { statistics by group, box plot, } \\
\text { histogram, Q-Q plot and } \\
\text { Shapiro-Wilk's test }\end{array}$ \\
\hline 2 & Correlation Analysis & $\operatorname{corrApp}()$ & $\begin{array}{l}\text { Simple correlation, correlation } \\
\text { Matrix, correlogram and } \\
\text { scatterplot }\end{array}$ \\
\hline 3 & $\begin{array}{l}\text { Compare Means: } \\
\text { Small samle tests }\end{array}$ & $\operatorname{tt} A p p()$ & $\begin{array}{l}\text { One sample t-test, two sample } \\
\text { unpaired t-test, paired t-test, } \\
\text { two sample unpaired Welch } \\
\text { t-test, F test, box plot, paired } \\
\text { Plot }\end{array}$ \\
\hline 4 & $\begin{array}{l}\text { Completely } \\
\text { Randomized Design }\end{array}$ & $\operatorname{crdApp}()$ & $\begin{array}{l}\text { One-way ANOVA (equal or } \\
\text { unequal replications), multiple } \\
\text { comparison test, boxplot and } \\
\text { barchart with confidence } \\
\text { interval }\end{array}$ \\
\hline 5 & $\begin{array}{l}\text { Field layout of } \\
\text { experiments }\end{array}$ & layoutApp() & $\begin{array}{l}\text { Field layouts of following } \\
\text { designs can be obtained: } \\
\text { completely randomized design } \\
\text { (CRD), randomized complete } \\
\text { block design (RCBD), } \\
\text { split-plot design, strip-plot } \\
\text { design, augmented RCBD }\end{array}$ \\
\hline 6 & $\begin{array}{l}\text { Randomized Block } \\
\text { Design }\end{array}$ & rbdApp () & $\begin{array}{l}\text { Two-way ANOVA, multiple } \\
\text { comparison test, boxplot and } \\
\text { barchart with confidence } \\
\text { interval }\end{array}$ \\
\hline
\end{tabular}

The package can be installed from CRAN as follows:

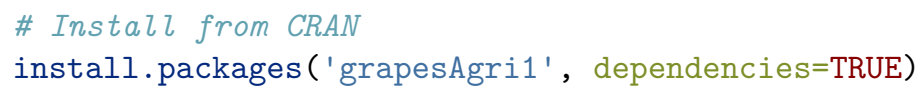

The development version can be installed from GitHub as follows: 
\# Install grapesAgri1 development version from Github using the code below: if (!require('devtools')) install.packages ('devtools')

devtools: : install_github("pratheesh3780/grapesAgri1")

\section{Package dependencies and details of functions used}

DescApp() function uses descr and stby functions of summarytools package (Comtois, 2021) to calculate summary statistics and summary statistics by group. knitr (Xie, 2021) and kableExtra(Zhu, 2021) packages were used to produce HTML tables. shapiro.test, qqnorm and qqline functions of stats package were used for the Test of Homogeneity of variance and obtaining $Q-Q$ plot. hist and boxplot of package graphics were used to obtain histogram and boxplot respectively. ggqqplot of package ggpubr(Kassambara, 2020) is also used to plot $\mathrm{Q}-\mathrm{Q}$ plot in the app.

CorrApp() function uses cor.test to calculate correlation. Correlation matrix is calculated using rcorr function in Hmisc package (Harrell Jr et al., 2021). Correlogram is obtained using corrplot function in corrplot(Wei \& Simko, 2021) package.

ttApp()function uses $t$.test function to calculate $t$ statistic. Descriptive statistics were calculated using stat.desc function of pastecs package. var.test function is used for F-test. ggboxplot function of ggpubr (Kassambara, 2020) package is used to draw boxplot. Paired plot is obtained using paired function of package PairedData(Champely, 2018).

$\operatorname{crdApp}()$ uses anova function of stats package to obtain one-way ANOVA. LSD.test,dun can. test and HSD. test functions of agricolae (de Mendiburu, 2020) package is used for multiple comparison test like LSD,DMRT and Tukey respectively. ggboxplot function of ggp ubr (Kassambara, 2020) package is used for boxplot. ggplot function of ggplot2(Wickham, 2016) is used for barchart with confidence interval.

layoutApp() uses design.crd, design.rcbd, design.dau, design.strip, design.split functions of package agricolae (de Mendiburu, 2020) to generate random layout of designs. Field layout were plotted using desplot function in desplot package (Wright, 2020).

$\operatorname{rbdApp}()$ uses anova function of stats package to obtain two-way ANOVA. LSD.test,du ncan.test and HSD.test functions of agricolae package (de Mendiburu, 2020) is used for multiple comparison test like LSD,DMRT and Tukey respectively. ggboxplot function of ggpubr package (Kassambara, 2020) is used for boxplot. ggplot function of ggplot2 (Wickham, 2016) is used for barchart with confidence interval.

\section{Usage}

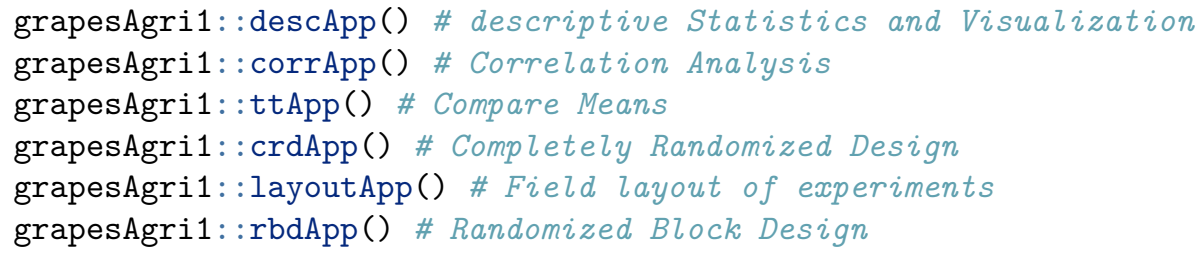

\section{Acknowledgements}

We wish to thank Kerala Agricultural University and Regional Agricultural Reaserch Station, Vellayani, Thiruvananthapuram for the financial support through the revolving fund scheme and observation trial. 


\section{References}

Champely, S. (2018). PairedData: Paired data analysis. https://CRAN.R-project.org/ package $=$ PairedData

Comtois, D. (2021). Summarytools: Tools to quickly and neatly summarize data. https: //CRAN.R-project.org/package=summarytools

de Mendiburu, F. (2020). Agricolae: Statistical procedures for agricultural research. https: //CRAN.R-project.org/package=agricolae

Harrell Jr, F. E., Charles Dupont, with contributions from, \& others., many. (2021). Hmisc: Harrell miscellaneous. https://CRAN.R-project.org/package=Hmisc

Kassambara, A. (2020). Ggpubr: 'ggplot2' based publication ready plots. https://CRAN. R-project.org/package $=$ ggpubr

Wei, T., \& Simko, V. (2021). R package "corrplot": Visualization of a correlation matrix. https://github.com/taiyun/corrplot

Wickham, H. (2016). ggplot2: Elegant graphics for data analysis. Springer-Verlag New York. ISBN: 978-3-319-24277-4

Wright, K. (2020). Desplot: Plotting field plans for agricultural experiments. https://CRAN. R-project.org/package $=$ desplot

Xie, Y. (2021). Knitr: A general-purpose package for dynamic report generation in r. https: //yihui.org/knitr/

Zhu, H. (2021). kableExtra: Construct complex table with 'kable' and pipe syntax. https: //CRAN.R-project.org/package=kableExtra 